\title{
Gadolinium Deposition in Deep Brain Structures: Relationship with Dose and Ionization of Linear Gadolinium-Based Contrast Agents
}

\author{
(D) H. Kang, (D) M. Hii, (D) M. Le, (D) R. Tam, (D) A. Riddehough, (D) A. Traboulsee, (D) Solind, (D) M.S. Freedman, and D.K.B. Li
}

\begin{abstract}
BACKGROUND AND PURPOSE: Dose-dependent association between hyperintensity in deep brain structures on unenhanced TIWIs and gadolinium-based contrast agent administrations has been demonstrated with subsequent histopathological confirmation of gadolinium deposition. Our aim was to determine whether greater exposure to linear gadolinium-based contrast agent administration is associated with higher signal intensity in deep brain structures on unenhanced T1-weighted MR imaging. Secondary objective was to compare signal intensity differences between ionic and nonionic linear gadolinium-based contrast agents.
\end{abstract}

MATERIALS AND METHODS: Subjects with secondary-progressive MS originally enrolled in a multicenter clinical trial were studied retrospectively. Eighty subjects (high-exposure cohort) received 9 linear gadolinium-based contrast agent administrations (30 nonionic/50 ionic) between week -4 and year 1 and a tenth administration by year 2. One hundred fifteen subjects (low-exposure cohort) received 2 administrations ( 40 nonionic/75 ionic) between week -4 and year 1 and a third administration by year 2 . Signal intensities were measured on unenhanced TWWls by placing sample-points on the dentate nucleus, globus pallidus, caudate, thalamus, pons, and white matter, and they were normalized using the following ratios: dentate/pons, globus pallidus/white matter, caudate/white matter, and thalamus/white matter.

RESULTS: Between week -4 and year 1 , subjects in the high-exposure cohort showed increased signal intensity ratios in all regions $(P<$ $.01)$, while the low-exposure cohort showed only an increase in the dentate nucleus $(P=.003)$. Between years 1 and 2 , when both cohorts received only 1 additional gadolinium-based contrast agent, no significant changes were observed. In the high-exposure cohort, significantly higher changes in signal intensity ratios were observed in subjects receiving linear nonionic than in those receiving linear ionic gadolinium-based contrast agents.

CONCLUSIONS: Hyperintensity in deep brain structures from gadolinium deposition is related to the number of doses and the type of linear gadolinium-based contrast agent (nonionic greater than ionic) administration.

ABBREVIATIONS: $\mathrm{CD}$ = caudate; $\mathrm{DN}=$ dentate nucleus; $\mathrm{GBCA}=$ gadolinium-based contrast agent; $\mathrm{GP}=$ globus pallidus; $\mathrm{SI}=$ signal intensity; $\mathrm{TH}=$ thalamus

G adolinium-based contrast agents (GBCAs) are frequently used in clinical MR imaging for their paramagnetic properties to shorten the $\mathrm{T} 1$ relaxation time of adjacent water protons

Received April 14, 2018; accepted after revision June 20.

From the Department of Radiology (H.K., M.L., R.T., S.K., D.K.B.L.), University of British Columbia MS/MRI Research Group (M.H., R.T., A.R., A.T., S.K., D.K.B.L.), Department of Medicine and Division of Neurology (A.T., S.K., D.K.B.L.), Department of Physics and Astronomy (S.K.), University of British Columbia, Vancouver, British Columbia, Canada; and Department of Medicine and Division of Neurology (M.S.F.), University of Ottawa and the Ottawa Hospital Research Institute, Ottawa, Canada.

Paper previously presented at: Annual Meeting of the American Society of Neuroradiology and the Foundation of the ASNR Symposium, June 2-7, 2018; Vancouver, British Columbia, Canada.

Please address correspondence to Heejun Kang, MD, Department of Radiology, UBC Hospital, 2211 Wesbrook Mall, Vancouver, BC V6T 2B5; e-mail:

tkang@alumni.ubc.ca; @tkang90

$\square$ Indicates article with supplemental on-line photos.

http://dx.doi.org/10.3174/ajnr.A5751 and increase the visibility of abnormal tissues, hence improving diagnostic value. ${ }^{1}$ However, free gadolinium is a toxic heavy metal in vivo and necessitates chelation to polyaminocarboxylic acid ligands to be excreted safely, primarily through the kidneys. Based on the chemical structures, there are 4 categories of chelating agents: macrocyclic ionic and nonionic, and linear ionic and nonionic. Stability and the ability to prevent free gadolinium from dissociating with the ligand differs for each type, with the macrocyclic being the most stable, followed by linear ionic, and linear nonionic being the least stable. ${ }^{2,3}$

In 2014, Kanda et $\mathrm{al}^{4}$ first reported that increased signal intensity (SI) in the dentate nucleus (DN) and globus pallidus (GP) on unenhanced T1WI was associated with repeat GBCA exposure. Subsequent cadaveric histopathologic studies showed that the T1 hyperintensity represented deposition of gadolinium in neuronal tissues. ${ }^{5,6}$ Since then, numerous studies have shown multiple ad- 


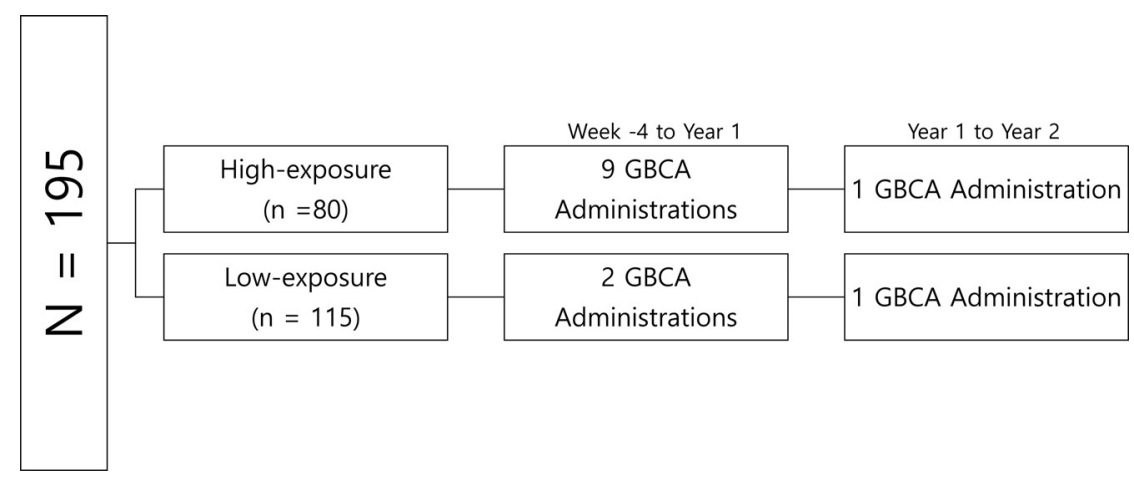

FIG 1. Gadolinium-based contrast agent administration schedule for high- and low-exposure cohorts.

ministrations of linear GBCAs to be associated with T1 hyperintensity in the deep brain structures. ${ }^{4,6-13}$ However, many of these studies did not have a control group and had a relatively small and heterogeneous sample population.

Furthermore, there is a paucity of literature comparing linear ionic with linear nonionic GBCAs. Ramalho et $\mathrm{al}^{14}$ was the only group at the time that directly compared the two and showed an increase in T1 hyperintensity with linear nonionic GBCA (gadodiamide) but not with linear ionic GBCA (gadobenate dimeglumine). Although other studies did demonstrate an increase in T1 hyperintensity with linear ionic GBCAs, direct comparison with linear nonionic GBCAs was not made. ${ }^{4,7,10}$

Our study used a cohort of 195 subjects with secondary-progressive MS who participated in a clinical trial and underwent 2 different contrast-enhanced MR imaging follow-up schedules (10 versus 3 GBCA administrations) over 2 years. Our goal was to determine whether multiple GBCA administrations were associated with increased $\mathrm{T} 1$ hyperintensity in deep brain structures on unenhanced T1WI and whether there was a dose relationship to these changes. Our secondary objective was to compare linear ionic with linear nonionic GBCAs to determine whether a difference could be observed in the degree of T1 SI change.

\section{MATERIALS AND METHODS Subjects}

MR imaging data were obtained from subjects with secondaryprogressive MS who were initially enrolled in a 2-year, randomized, double-blind, placebo-controlled trial assessing the efficacy of dirucotide (MBP8298) (clinicaltrials.gov NCT00869726 and ISRCTN98373474). ${ }^{15}$ Six hundred twelve subjects were recruited from 47 centers in 10 countries.

Of the 612 subjects, 553 completed the trial and had usable MR imaging datasets. Ethics committees at each site approved the study, and all subjects signed written informed consents before protocol-required procedures. The details of the design, inclusion, and exclusion criteria and results of the 2-year study were previously reported. ${ }^{15}$ There were no significant differences regarding clinical or MR imaging outcomes between the treatment and placebo cohorts, except the cumulative number of new and enlarging T2 lesions that favored the placebo cohort $(P=.03) .{ }^{15}$ Therefore, the subjects in both treatment and placebo arms were pooled together to be analyzed in the current study. MR imaging data were centrally analyzed by our research institution (UBC MS/MRI Research Group).

Exclusion criteria for the MR imaging data of this retrospective study were the absence of unenhanced T1WI, unsatisfactory image quality due to artifacts, and the presence of visible lesions in both the left and right $\mathrm{DN}$, thalamus (TH), GP, caudate (CD), and/or frontal white matter in any of the time points. If the MR imaging data met the exclusion criteria, then all imaging studies for the same subject at other time points were removed from the study analysis. In addition, subjects who were administered macrocyclic GBCAs (the use of these agents was uncommon during the study period) and/or were scanned on a different MR imaging scanner for any of their follow-up studies were excluded.

\section{GBCA Administration and Type}

The original study population followed 2 distinct contrast-enhanced MR imaging follow-up schedules: In the first 100 subjects (high-exposure cohort), 2 MRIs (week -4, week 0) were performed before the first dose of study medication, followed by further MRIs at weeks 4, 8, and 12. Another MR imaging was then performed immediately before the next dose (week 26), followed by 3 further MRIs at weeks 30, 34, and 38. Annual MRIs were then performed at years 1 and 2 (weeks 52 and 104). Overall, for the year 1 pre-GBCA assessment, 9 GBCA injections were given between week -4 and year 1 , and for the year 2 pre-GBCA assessment, an additional GBCA was administered (from the year $1 \mathrm{MR}$ imaging). The next 453 subjects (low-exposure cohort) had 2 MRIs performed before the first dose of medication (weeks -4 and 0) with annual MRIs at years 1 and 2 (weeks 52 and 104). Therefore, for the year 1 pre-GBCA assessment, 2 GBCA injections had been given, and similarly, for the year 2 pre-GBCA assessment, an additional GBCA injection was administered (from the year 1 MR imaging) (Fig 1). A random sample of the subjects in the low-exposure cohort was selected proportionately from each high-exposure cohort center. Subjects for this study received a standard single dose of either a linear nonionic agent (gadodiamide, Omniscan; GE Healthcare, Piscataway, New Jersey) or a linear ionic agent (gadopentetate dimeglumine, Magnevist; Bayer HealthCare Pharmaceuticals, Wayne, New Jersey), depending on the center preference. The same GBCA was used throughout the duration of the study.

\section{MR Imaging Protocol, Processing, and Analysis}

Studies were performed with either $1 \mathrm{~T}, 1.5 \mathrm{~T}$, or $3 \mathrm{~T}$ MR imaging scanners (Table 1) using a standardized imaging protocol with whole-brain coverage, 3-mm contiguous axial proton density, $\mathrm{T} 2 \mathrm{WI}$, and pre- and postcontrast $(0.1 \mathrm{mmol} / \mathrm{kg}$ gadolinium with a 5-minute delay) fast/turbo spin-echo T1WI (TE, 9-20 ms; TR, 600-800 ms; and slice gap, $0 \mathrm{~mm}$ ). Changes to the MR imaging protocol were not permitted during the trial period.

Group-wise registration was performed using the T2WIs for 
Table 1: Demographics

\begin{tabular}{|c|c|c|c|}
\hline Characteristic & High-Exposure Group ( $n=80$ ) & Low-Exposure Group ( $n=115)$ & $P$ Value \\
\hline Age $(y r)^{a}$ & $51 \pm 8(34-64)$ & $52 \pm 8(27-82)$ & NS \\
\hline \multicolumn{4}{|l|}{ Sex } \\
\hline Female & $52(65 \%)$ & 78 (68\%) & \\
\hline Male & $28(35 \%)$ & $37(32 \%)$ & \\
\hline Weight $(\mathrm{kg})^{\mathrm{a}}$ & $72 \pm 16(48-120)$ & $71 \pm 15(48-117)$ & \\
\hline \multicolumn{4}{|l|}{ Gadolinium type } \\
\hline Gadodiamide & $30(37.5 \%)$ & $40(34.8 \%)$ & \\
\hline Gadopentetate dimeglumine & $50(62.5 \%)$ & $75(65.2 \%)$ & \\
\hline \multicolumn{4}{|l|}{ Treatment assignment } \\
\hline MBP8298 & $41(51 \%)$ & $57(50 \%)$ & \\
\hline Placebo & $39(49 \%)$ & $58(50 \%)$ & \\
\hline EDSS at week $-4^{a}$ & $5.57 \pm 1.03(3-6.5)$ & $5.34 \pm 1.13(3-6.5)$ & NS \\
\hline EDSS at year $2^{\mathrm{a}}$ & $5.61 \pm 1.00(3.5-6.5)$ & $5.66 \pm 1.19(2-7.5)$ & NS \\
\hline \multirow{2}{*}{ Disease duration $(y r)^{a}$} & $13.6 \pm 5.5(4-25)$ & $11.7 \pm 5.1(2-27)$ & NS \\
\hline & $(n=65)$ & $(n=84)$ & \\
\hline \multicolumn{4}{|l|}{ Magnet strength } \\
\hline $1 T$ & $0(0 \%)$ & $4(3 \%)$ & \\
\hline $1.5 \mathrm{~T}$ & $58(73 \%)$ & $93(81 \%)$ & \\
\hline $3 T$ & $22(27 \%)$ & $18(16 \%)$ & \\
\hline
\end{tabular}

Note:-EDSS indicates Expanded Disability Status Scale; NS, not significant.

${ }^{a}$ Data are means \pm SD (range).

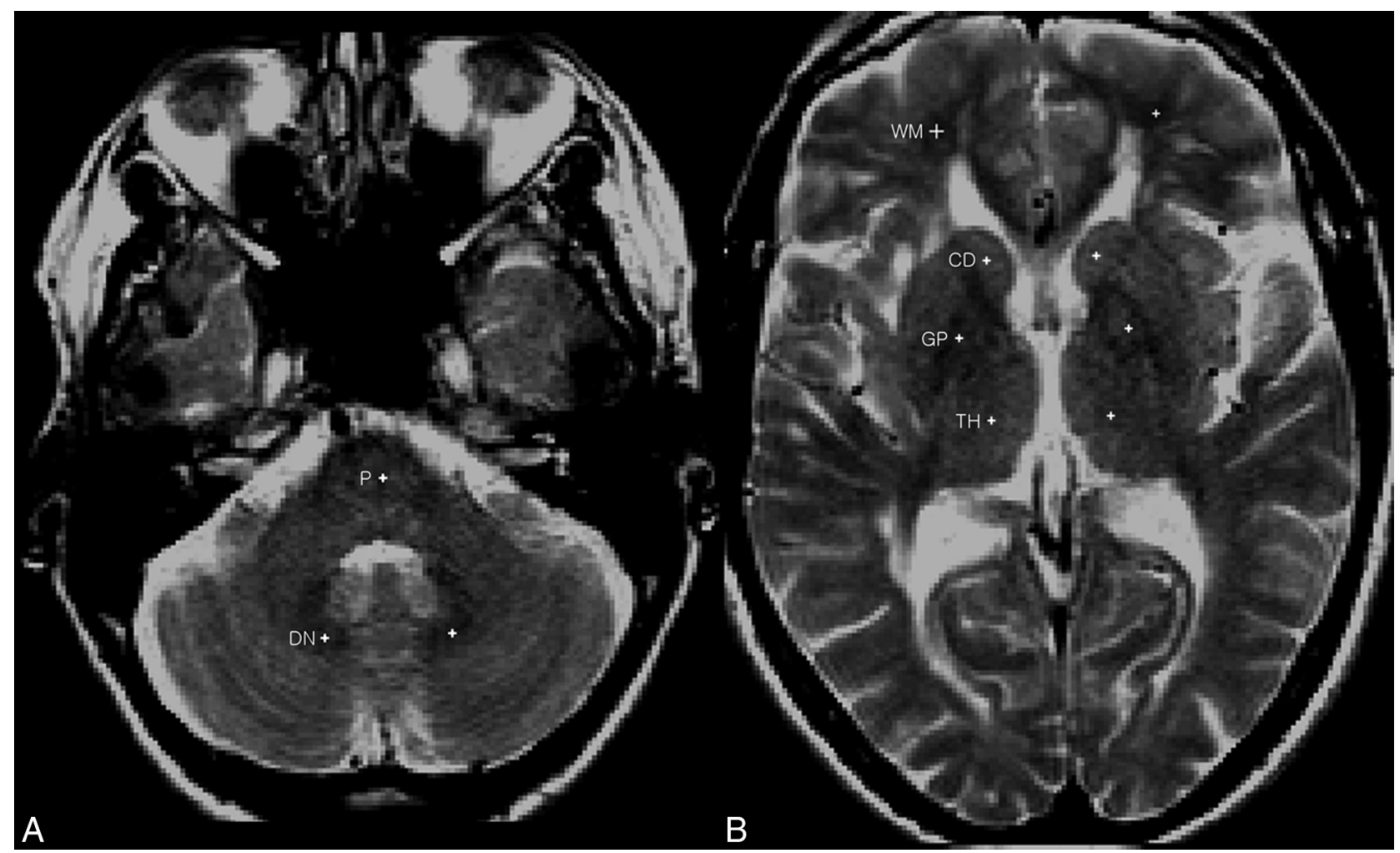

FIG 2. A, T2-weighted spin-echo images at the level of the posterior fossa with sample-points (white cross) placed at the center of the dentate and pons (P). B, T2-weighted spin-echo images at the level of the third ventricle with sample-points (white cross) placed at the center of the caudate, thalamus, globus pallidus, and white matter.

all subjects across 3 time points (week -4 , year 1, and year 2) to ensure that the exact same location was measured across all time points. The sample-points $(1 \times 1$ pixel; 1 pixel $=0.937 \mathrm{~mm})$ were manually placed on the T2WIs by a single reader (M.L.) blinded to cohort assignment at our research institution. T2WIs were chosen instead of T1WIs to blind the reader from any visibly obvious T1 hyperintensity that may bias sample-point placement. In addition, T2WIs enabled the reader to identify the deep brain structures more easily. Sample-points were placed at the center of the left DN, left GP, left TH, left CD head, central pons, and left frontal WM (Fig 2). Normal-appearing WM that was free of any visible lesions was used. If the left side could not be assessed due to the presence of artifacts or visible lesions, then the right side was used. If the deep brain structures were unclear on T2WIs, the same section position of proton density images was used to guide placement. The final sample-point position was confirmed by a second reader (H.K.), and the sample-point measurement was conducted once. The sample-points were then mapped onto the 


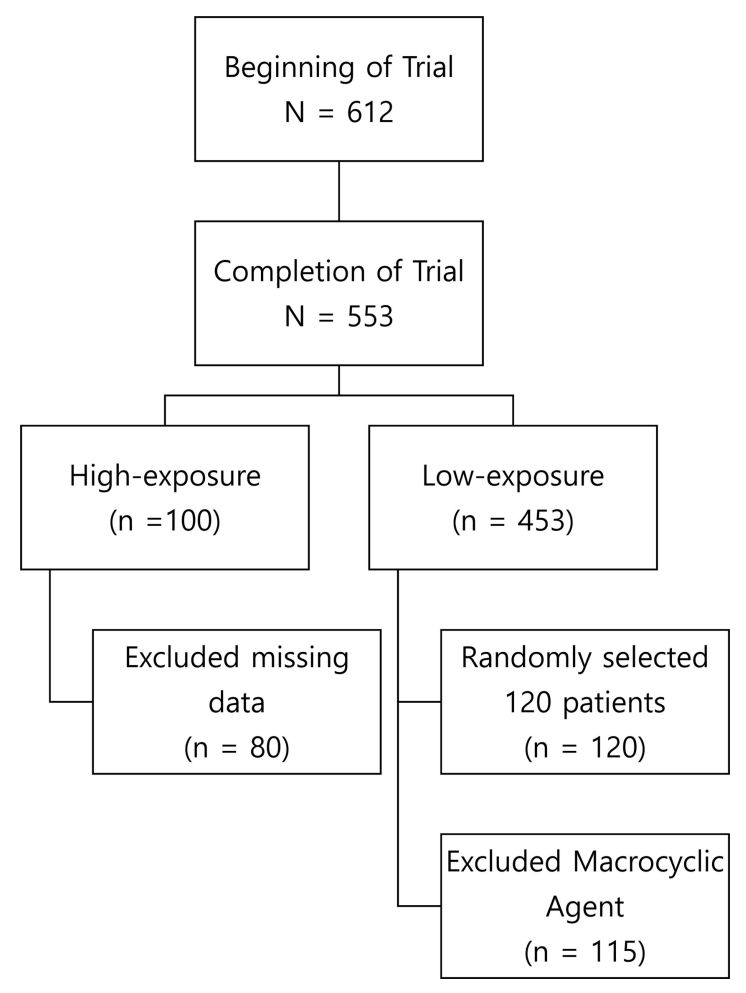

FIG 3. Patient disposition.

original precontrast (ie, unenhanced) T1WIs, and mean T1 SIs were obtained. Normalization of the mean T1 SI was performed by calculating the following ratios: DN/pons, GP/WM, CD/WM, and TH/WM.

\section{Statistical Analysis}

Statistical analysis was performed with a commercially available medical statistical package (GraphPad Prism software; GraphPad Software, San Diego, California). The two-sample paired $t$ test was used to evaluate the SI changes in the DN/pons, GP/WM, TH/WM, and CD/WM between week -4 and year 1 , years 1 and 2 , and week -4 and year 2 for the high-exposure and low-exposure cohorts. The two-sample unpaired $t$ test was used to compare the SI changes in the DN/pons, GP/WM, TH/WM, and CD/WM between the subjects receiving linear nonionic versus the subjects receiving linear ionic GBCAs in the high-exposure cohort from week -4 to year 2 . For all statistical tests, the level of significance was set at $P \leq .05$.

\section{RESULTS}

Of the 100 subjects in the high-exposure cohort, 19 had missing MR imaging data at different time points and 1 subject had a major scanner change during follow-up, hence they were excluded. Overall, 80 subjects had complete MR imaging scan sets that also met the acceptance criteria for T1 SI analysis. Of the 453 subjects in the low-exposure cohort, 120 were randomly selected for analysis in which each high-exposure cohort center was proportionally represented (Fig 3). Five subjects from the low-exposure cohort were administered a macrocyclic agent and were excluded from the analysis. The high- and low-exposure cohorts had similar baseline characteristics (Table 1).
In the high-exposure cohort, 30 subjects received a linear nonionic agent (gadodiamide, Omniscan), while 50 subjects received a linear ionic agent (gadopentetate dimeglumine, Magnevist). For the low-exposure cohort, 40 subjects received a linear nonionic agent (gadodiamide, Omniscan) and 75 subjects received a linear ionic agent (gadopentetate dimeglumine, Magnevist). In the high-exposure cohort, the left DN or the left WM for 2 subjects was replaced with the right side for all 3 time points due to the presence of a visible lesion. In the low-exposure group, 1 subject's left WM showed a visible lesion and the right side was used instead for all 3 time points.

Between week -4 and year 1 , there were significant absolute increases in all measured SI ratios (mean \pm SD) in the highexposure group (DN/pons: $0.04 \pm 0.09$ [relative increase of $+4 \%$ ]; GP/WM: $0.05 \pm 0.09$ [relative increase of $+5 \%$ ]; CD/ WM: $0.03 \pm 0.08$ [relative increase of $+3 \%$ ]; and TH/WM: $0.03 \pm 0.09$ [relative increase of $+3 \%$ ]) (Table 2 and Fig 4), while only the DN/pons ratio showed a significant increase in the lowexposure group of $0.05 \pm 0.17$ (relative increase of $+5 \%$ ) (Table 3 ). Between years 1 and 2, when both cohorts received only 1 additional GBCA injection, no significant difference was observed in all SI ratios (Tables 2 and 3). Overall, from week -4 to year 2, significant SI increases were seen in all deep brain structures in the high-exposure cohort, but only the $\mathrm{DN} /$ pons ratio continued to show a significant increase in the low-exposure group (Tables 2 and 3). In the high-exposure group, 1 outlier was identified in the GP/WM and CD/WM SI measurements (On-Line Fig 1); and in the low-exposure group, 6 outliers in the DN/pons and 1 outlier in the GP/WM, CD/WM, and TH/WM SI measurements were demonstrated (On-Line Fig 2). The overall results and statistical significance remained consistent when the analysis was repeated with the outliers excluded.

Subgroup analysis of the subjects in the high-exposure cohort who received linear nonionic GBCA showed a significant increase in all SI ratios from week -4 to year 2 (Table 4 ). In comparison, for subjects who received a linear ionic GBCA, a significant increase was detected for only the $\mathrm{DN} /$ pons and $\mathrm{CD} / \mathrm{WM}$ ratios (Table 4). There was more than a 2 -fold difference in the DN/pons ratios, where the linear nonionic GBCA group demonstrated an average of $0.08 \pm 0.09(7.8 \%)$ increase, compared with $0.03 \pm$ $0.09(3.3 \%)$ in the linear ionic group $(P=.02)$.

\section{DISCUSSION}

There was a dose-dependent relationship between the number of linear GBCA administrations and the SI ratio increase in deep brain structures on unenhanced T1WI. After receiving 9 GBCA administrations, the high-exposure cohort demonstrated significant increases in all measured SI ratios (DN, GP, CD, and TH), while only the DN showed a significant increase in the low-exposure group after 2 GBCA administrations during the same period. This finding signifies that the larger number of GBCA administrations was a major factor in a greater number of deep brain structures demonstrating significant SI ratio increases in the highexposure cohort compared to the low-exposure cohort, supporting a dose relationship. This result is in keeping with previous retrospective studies that have also demonstrated a greater increase in measured SI ratios to be related to multiple linear GBCA 
Table 2: Absolute and relative increase in signal intensity ratios for the high-exposure cohort ${ }^{\mathrm{a}}$

\begin{tabular}{lcccccc}
\hline & $\boldsymbol{\Delta}$ Week -4 to Year 1 & $\boldsymbol{P}$ Value & $\boldsymbol{\Delta}$ Year 1 to Year 2 & $\boldsymbol{P}$ Value & $\boldsymbol{\Delta}$ Week $-\mathbf{4}$ to Year 2 & $\boldsymbol{P}$ Value \\
\hline L. DN & $0.04 \pm 0.09(4 \%)$ & $<.001$ & $0.01 \pm 0.09(1 \%)$ & NS & $0.05 \pm 0.09(5 \%)$ & $<.001$ \\
L. GP & $0.05 \pm 0.09(5 \%)$ & $<.001$ & $-0.02 \pm 0.11(-1 \%)$ & NS & $0.03 \pm 0.10(3 \%)$ & .005 \\
L. CD & $0.03 \pm 0.08(3 \%)$ & $<.001$ & $0 \pm 0.11(0 \%)$ & NS & $0.03 \pm 0.09(3 \%)$ & .005 \\
L. TH & $0.03 \pm 0.09(3 \%)$ & .01 & $0 \pm 0.11(0 \%)$ & NS & $0.03 \pm 0.09(3 \%)$ & .01 \\
\hline
\end{tabular}

Note:-L. indicates left; NS, not significant.

${ }^{a}$ Data are absolute (mean \pm SD) and relative $(\%)$ increases.

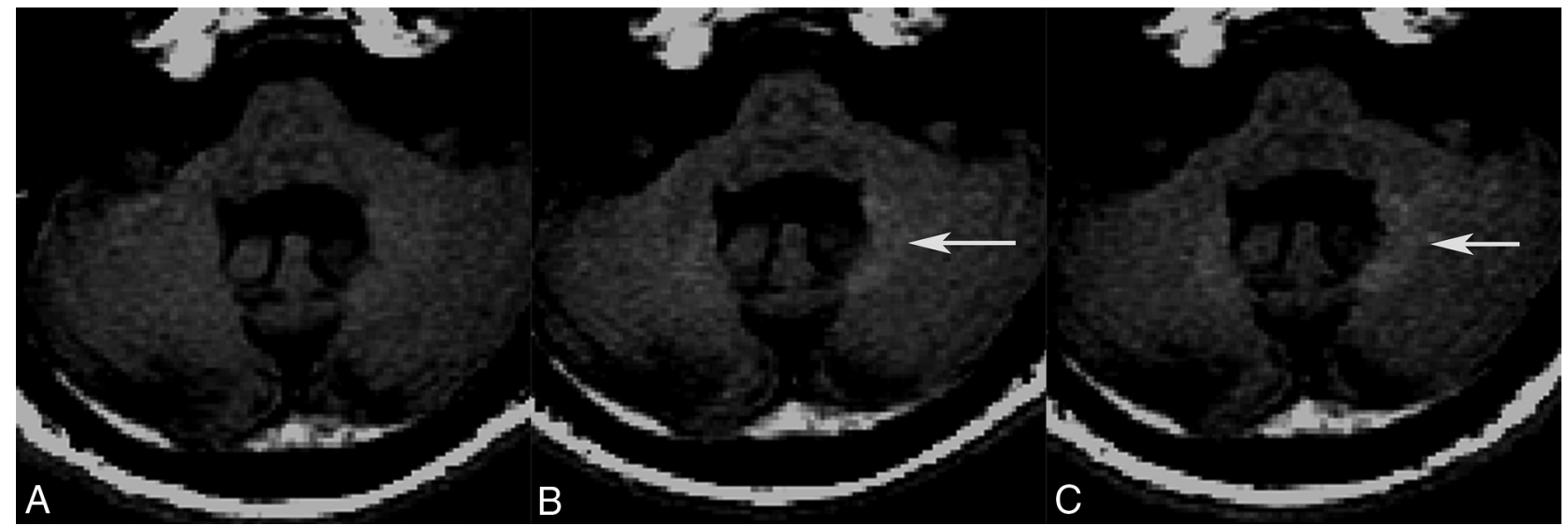

FIG 4. Unenhanced axial fast spin-echo T1-weighted MR images of a 48-year-old man in the high-exposure cohort who received linear nonionic GBCA. Images were obtained at week $-4(A)$, year $1(B)$, and year $2(C)$. A significant increase in 71 signal intensity is visualized in the dentate by year 1 (arrow), which persisted to year 2 (arrow).

Table 3: Absolute and relative increase in signal intensity ratios for the low-exposure cohort $^{\mathrm{a}}$

\begin{tabular}{lcccccc}
\hline & $\boldsymbol{\Delta}$ Week $-\mathbf{4}$ to Year 1 & $\boldsymbol{P}$ Value & $\boldsymbol{\Delta}$ Year 1 to Year 2 & $\boldsymbol{P}$ Value & $\boldsymbol{\Delta}$ Week $-\mathbf{4}$ to Year 2 & $\boldsymbol{P}$ Value \\
\hline L. DN & $0.05 \pm 0.17(5 \%)$ & .003 & $0 \pm 0.20(0 \%)$ & NS & $0.04 \pm 0.15(4 \%)$ & 003 \\
L. GP & $0.01 \pm 0.09(1 \%)$ & NS & $0 \pm 0.17(0 \%)$ & NS & $0 \pm 0.18(0 \%)$ & NS \\
L. CD & $0.01 \pm 0.09(1 \%)$ & NS & $0 \pm 0.22(0 \%)$ & NS & $0 \pm 0.21(0 \%)$ & NS \\
L. TH & $0 \pm 0.09(0 \%)$ & NS & $0.01 \pm 0.18(1 \%)$ & NS & $0.01 \pm 0.19(1 \%)$ & NS \\
\hline
\end{tabular}

Note:- L. indicates left; NS, not significant.

${ }^{a}$ Data are absolute (mean \pm SD) and relative (\%) increases.

Table 4: Absolute and relative increase in signal intensity ratios in subjects who received linear nonionic and linear ionic GBCA in the high-exposure cohort ${ }^{\mathrm{a}}$

\begin{tabular}{lcccc}
\hline & \multicolumn{4}{c}{$\boldsymbol{\Delta}$ Week $-\mathbf{4}$ to Year $\mathbf{2}$} \\
\cline { 2 - 5 } & Linear Nonionic & $\boldsymbol{P}$ Value & Linear lonic & $\boldsymbol{P}$ Value \\
\hline L. DN & $0.08 \pm 0.09(8 \%)$ & $<.001$ & $0.03 \pm 0.09(3 \%)$ & .01 \\
L. GP & $0.06 \pm 0.06(6 \%)$ & $<.001$ & $0.02 \pm 0.11(2 \%)$ & NS \\
L. CD & $0.03 \pm 0.06(3 \%)$ & .03 & $0.03 \pm 0.10(3 \%)$ & .04 \\
L. TH & $0.04 \pm 0.06(4 \%)$ & .001 & $0.02 \pm 0.10(2 \%)$ & NS \\
\hline
\end{tabular}

Note:-L. indicates left; NS, not significant.

${ }^{a}$ Data are absolute (mean $\pm \mathrm{SD}$ ) and relative (\%) increases.

injections. ${ }^{4,6,7,9-14,16-20}$ However, both the low- and the high-exposure cohorts had a significant-but-similar increase in the DN within the first year, and a clear dose effect was not seen for this structure. The DN is known to be more sensitive to gadolinium accumulation, and we could be seeing a dose-saturation effect in this structure, though the exact nature is not elucidated by our methodology. The importance of the number of GBCA administrations and its relationship with the T1 hyperintensities is further supported from the results between years 1 and 2, when no SI ratio changes were seen when the same high- and low-exposure cohorts received only 1 GBCA administration during the same period. We suspect that the increases in SI ratios were too minute and our measurement technique was not sensitive in detecting the changes.
The subgroup analysis in the highexposure cohort comparing the linear ionic GBCA (gadopentetate dimeglumine) and the linear nonionic GBCA (gadodiamide) showed that the linear nonionic GBCA had the greatest increase in $\mathrm{T} 1$ hyperintensity in the deep brain structures. In particular, SI increases were demonstrated in all deep brain structures with the linear nonionic GBCA, whereas only the DN and CD demonstrated SI increase for the linear ionic GBCA. The DN demonstrated more than a 2 -fold difference between the 2 classes of GBCAs, with an $8 \%$ increase in the linear nonionic GBCA compared with a $3 \%$ increase in the linear ionic GBCA $(P=.02)$. These findings demonstrate in vivo the in vitro results by Frenzel et $\mathrm{al}^{2}{ }^{2}$ who showed that the linear nonionic GBCAs was less stable than the linear ionic GBCAs. In their study, approximately $20 \%$ of the gadolinium of the linear nonionic GBCAs was released after 15 days of incubation in human serum, compared with $1 \%-2 \%$ of the gadolinium of the linear ionic GBCAs. ${ }^{2}$ No gadolinium was released with the macrocyclic GBCAs. ${ }^{2}$ Overall, these findings are in support of the hypothesis that the propensity of a GBCA to cause hyperintensity depends on the specific stability of the GBCA. ${ }^{21}$

AJNR Am J Neuroradiol 39:1597-1603 Sep 2018 www.ajnr.org

1601 
Recent studies in rats propose that the penetration of GBCAs into the brain may occur through the blood-CSF barrier. ${ }^{22,23}$ Once inside the brain tissue, the chemical stability and tendency of different GBCAs to dechelate may play an important role in the deposition of gadolinium in brain tissues. A new study by Frenzel et $\mathrm{al}^{24}$ showed that after administration of linear ionic and linear nonionic GBCAs, a large portion of the gadolinium was detected in rat brain tissues as insoluble fractions or bound to soluble macromolecules, both presumed responsible for increased T1 SI due to their high relaxivity. These molecules were not found for macrocyclic GBCAs, which were exclusively detected in the soluble fraction, likely in their intact form, and demonstrated ongoing excretion. ${ }^{24}$ However, more research is required to determine the clinical consequences of gadolinium deposition in either the chelated or dechelated form.

As demonstrated on a histopathologic study, the DN appears to be the most sensitive structure for detecting the T1 SI increase. ${ }^{6}$ In our study, even after only 2 GBCA administrations, an increase in T1 hyperintensity was detected in the DN. One possible explanation is that the DN, along with other deep brain structures, is more susceptible to transmetalation, which allows dechelated gadolinium to form high-relaxivity macromolecules, as described above. $^{25}$

Our study has several limitations. First, because the number of subjects receiving macrocyclic GBCA was small, this study could not examine the difference between the use of linear and macrocyclic GBCAs. Second, as with most studies on GBCA administration, the history of prior GBCA administrations and use of other agents could not be determined. Our study shows that there was at least a further increase in the SI during our observation window. Third, due to the multicenter nature of the study, several different MR imaging scanners were used, which may have introduced variations among scans, subjects, and scanners. Effort was made to minimize this issue by proportionately representing each center in both the high- and low-exposure cohorts. In addition, each follow-up MR imaging study was performed using the same MR imaging machine in the same center for each subject, and the SI was normalized with the reference standard extracted from the same image slice (pons and WM). The DN/pons ratio was chosen because McDonald et $\mathrm{al}^{6}$ demonstrated in postmortem tissue 23fold less gadolinium in the pons than in the DN. The WM tract was chosen for the CD, GP, and TH because it was hypothesized that the WM would accumulate less gadolinium than the gray matter structures, such as the TH. Fourth, the sample-point size used for the study measured $1 \times 1$ pixel, which likely introduced noise and fluctuations in the SI measurements. However, the small size was chosen to improve the accuracy of the sample-point placement on small structures such as the DN and CD, which was important when the sample-points were mapped from T2WI to T1WI. Furthermore, the degree of SI changes between the highand low-exposure cohorts was large enough to overcome this limitation and demonstrated significant and relatively consistent results. Fifth, the study population had a pre-existing neurologic disease (MS), which may have confounded the results. However, the degree of interference is thought to be minimal because both cohorts had the same diagnosis with similar disease duration and Expanded Disability Status Scale scores without other po- tential confounding disease processes. Last, the physical, cognitive, and behavioral outcomes were not analyzed in this study; however, these are the subject of a current investigation.

\section{CONCLUSIONS}

As little as 2 doses of GBCAs can result in an increased T1 SI in the $\mathrm{DN}$ that persists for at least 1 year after administration. T1 hyperintensity in the GP, CD, and TH is evident with greater cumulative doses. The degree of increase is related to the location (the DN being the most sensitive), the number of GBCA administrations, and the class of GBCA, with linear nonionic having the greater deposition.

Disclosures: Roger Tam—RELATED: Other: Jefferson Mooney, Comments: Mr Mooney donated $\$ 10,000$ to support trainees in multiple sclerosis research at the University of British Columbia. This donation had no other restrictions on its use, and we used it to support Megan $\mathrm{He}$, an undergraduate intern who worked on the technical aspects of the project*; UNRELATED: Grants/Grants Pending: Natural Sciences and Engineering Research Council of Canada, Comments: grant support from Natural Sciences and Engineering Research Council*; Other: Roche, Genzyme, Comments: Our group participates in sponsored clinical studies and clinical trials, which are unrelated to the submitted work.* Anthony Traboulsee-UNRELATED: Other: Consortium of Multiple Sclerosis Centers, Comments: honoraria to develop and disseminate MRI guidelines for diagnosing and monitoring patients with MS, including the role of gadolinium. Shannon Kolind-UNRELATED: Consultancy: Acorda Therapeutics; Grants/Grants Pending: Sanofi, Roche. Mark S. FreedmanUNRELATED: Consultancy: Actelion Pharmaceutical, Bayer Healthcare, Biogen, Chugai Pharmaceutical, Clene, EMD Serono Canada, Genzyme, Merck Serono, MedDay, Novartis, Roche, Sanofi, Teva Pharmaceutical Industries, Comments: consultant, ad board, steering committee; Grants/Grants Pending: Genzyme, Comments: Investigator Sponsored Studies (ISS)*; Payment for Lectures Including Service on Speakers Bureaus: Genzyme, Sanofi, Merck, Actelion, Biogen, EMD, Teva Pharmaceutical Industries; Payment for Development of Educational Presentations: Roche. David K.B. Li-UNRELATED: Consultancy: Vertex Pharmaceuticals, Opexa Therapeutics, Adelphi Group, Comments: I have been a consultant for Vertex Pharmaceuticals and served on the Data and Safety Advisory Board for Opexa Therapeutics and the Scientific Advisory Board for the Adelphi Group; Grants/Grants Pending: Genzyme, Hoffmann-la Roche, Merck Serono. Perceptive/Novartis, Sanofi-Aventis, Comments: I am the Emeritus Director of the University of British Columbia MS/MRI Research Group, which has received contracts to provide the central MRI analysis for MS clinical trials*; Payment for Lectures Including Service on Speakers Bureaus: Academy of Health Care Learning, Biogen Idec, Consortium of Multiple Sclerosis Centers, Novartis, Sanofi Genzyme, and Teva Pharmaceutical Industries; Payment for Development of Educational Presentations: Academy of Health Care Learning, Comments: Webinar. *Money paid to the institution in Canadian dollars.

\section{REFERENCES}

1. Hao D, Ai T, Goerner F, et al. MRI contrast agents: basic chemistry and safety. J Magn Reson Imaging 2012;36:1060-71 CrossRef Medline

2. Frenzel T, Lengsfeld P, Schirmer $H$, et al. Stability of gadoliniumbased magnetic resonance imaging contrast agents in human serum at $37^{\circ} \mathrm{C}$. Invest Radiol 2008;43:817-28 CrossRef Medline

3. Tweedle MF, Wedeking P, Kumar K. Biodistribution of radiolabeled, formulated gadopentetate, gadoteridol, gadoterate, and gadodiamide in mice and rats. Invest Radiol 1995;30:372-80 CrossRef Medline

4. Kanda T, Ishii $\mathrm{K}$, Kawaguchi $\mathrm{H}$, et al. High signal intensity in the dentate nucleus and globus pallidus on unenhanced T1weighted MR images: relationship with increasing cumulative dose of a gadolinium-based contrast material. Radiology 2014; 270:834-41 CrossRef Medline

5. Murata N, Gonzalez-Cuyar LF, Murata K, et al. Macrocyclic and other non-group 1 gadolinium contrast agents deposit low levels of gadolinium in brain and bone tissue: preliminary results from 9 patients with normal renal function. Invest Radiol 2016;51:447-53 CrossRef Medline

6. McDonald RJ, McDonald JS, Kallmes DF, et al. Intracranial gadolin- 
ium deposition after contrast-enhanced MR imaging. Radiology 2015;275:772-82 CrossRef Medline

7. Kanda T, Osawa M, Oba $\mathrm{H}$, et al. High signal intensity in dentate nucleus on unenhanced T1-weighted MR images: association with linear versus macrocyclic gadolinium chelate administration. $R a-$ diology 2015;275:803-09 CrossRef Medline

8. Quattrocchi CC, Mallio CA, Errante Y, et al. Gadodiamide and dentate nucleus $\mathrm{T} 1$ hyperintensity in patients with meningioma evaluated by multiple follow-up contrast-enhanced magnetic resonance examinations with no systemic interval therapy. Invest Radiol 2015; 50:470-72 CrossRef Medline

9. Errante Y, Cirimele V, Mallio CA, et al. Progressive increase of T1 signal intensity of the dentate nucleus on unenhanced magnetic resonance images is associated with cumulative doses of intravenously administered gadodiamide in patients with normal renal function, suggesting dechelation. Invest Radiol 2014;49: 685-90 CrossRef Medline

10. Radbruch A, Weberling LD, Kieslich PJ, et al. Gadolinium retention in the dentate nucleus and globus pallidus is dependent on the class of contrast agent. Radiology 2015;275:783-91 CrossRef Medline

11. Cao $Y$, Huang DQ, Shih G, et al. Signal change in the dentate nucleus on T1-weighted MR images after multiple administrations of gadopentetate dimeglumine versus gadobutrol. $A J R$ Am J Roentgenol 2016;206:414-19 CrossRef Medline

12. Weberling LD, Kieslich PJ, Kickingereder P, et al. Increased signal intensity in the dentate nucleus on unenhanced T1-weighted images after gadobenate dimeglumine administration. Invest Radiol 2015;50:743-48 CrossRef Medline

13. Radbruch A, Weberling LD, Kieslich PJ, et al. Intraindividual analysis of signal intensity changes in the dentate nucleus after consecutive serial applications of linear and macrocyclic gadolinium-based contrast agents. Invest Radiol 2016;51:683-90 CrossRef Medline

14. Ramalho J, Castillo M, AlObaidy M, et al. High signal intensity in globus pallidus and dentate nucleus on unenhanced T1-weighted MR images: evaluation of two linear gadolinium-based contrast agents. Radiology 2015;276:836-44 CrossRef Medline

15. Freedman MS, Bar-Or A, Oger J, et al; MAESTRO-01 Investigators. A phase III study evaluating the efficacy and safety of MBP8298 in secondary progressive MS. Neurology 2011;77:1551-60 CrossRef Medline

16. Adin ME, Kleinberg L, Vaidya D, et al. Hyperintense dentate nuclei on T1-weighted MRI: relation to repeat gadolinium administration. AJNR Am J Neuroradiol 2015;36:1859-65 CrossRef Medline

17. Flood TF, Stence NV, Maloney JA, et al. Pediatric brain: repeated exposure to linear gadolinium-based contrast material is associated with increased signal intensity at unenhanced T1-weighted MR imaging. Radiology 2017;282:222-28 CrossRef Medline

18. Hu HH, Pokorney A, Towbin RB, et al. Increased signal intensities in the dentate nucleus and globus pallidus on unenhanced T1-weighted images: evidence in children undergoing multiple gadolinium MRI exams. Pediatr Radiol 2016;46:1590-98 CrossRef Medline

19. Ramalho J, Semelka RC, Ramalho M, et al. Gadolinium-based contrast agent accumulation and toxicity: an update. AJNR Am J Neuroradiol 2016;37:1192-98 CrossRef Medline

20. Zhang Y, Cao Y, Shih GL, et al. Extent of signal hyperintensity on unenhanced T1-weighted brain MR images after more than 35 administrations of linear gadolinium-based contrast agents. Radiology 2017;282:516-25 CrossRef Medline

21. Radbruch A. Are some agents less likely to deposit gadolinium in the brain? Magn Reson Imaging 2016;34:1351-54 CrossRef Medline

22. Jost G, Frenzel T, Lohrke J, et al. Penetration and distribution of gadolinium-based contrast agents into the cerebrospinal fluid in healthy rats: a potential pathway of entry into the brain tissue. Eur Radiol 2017;27:2877-85 CrossRef Medline

23. Öner AY, Barutcu B, Aykol Ş, et al. Intrathecal contrast-enhanced magnetic resonance imaging-related brain signal changes: residual gadolinium deposition? Invest Radiol 2017;52:195-97 CrossRef Medline

24. Frenzel T, Apte C, Jost G, et al. Quantification and assessment of the chemical form of residual gadolinium in the brain after repeated administration of gadolinium-based contrast agents: comparative study in rats. Invest Radiol 2017;52:396-404 CrossRef Medline

25. Gulani V, Calamante F, Shellock FG, et al; International Society for Magnetic Resonance in Medicine. Chelated or dechelated gadolinium deposition: authors' reply. Lancet Neurol 2017;16:955-56 CrossRef Medline 